\title{
Calidad de vida y caracterización de las personas con Enfermedad Renal Crónica trasplantadas
}

\author{
Claudia Patricia Cantillo-Medina ${ }^{1}$, Liseth Fabiana Sánchez-Castro², Angie Melissa Ramírez-Guerrero², Marly \\ Daniela Muñoz-Bolaños², Hernán Felipe Quintero-Penagos², Stephany Valentina Cuero-Montaño² \\ ${ }^{1}$ Departamento de Enfermería. Facultad de Salud. Universidad Surcolombiana. Neiva. Huila. Colombia \\ ${ }^{2}$ Programa de Enfermería. Facultad de Salud. Universidad Surcolombiana. Neiva. Huila. Colombia
}

Como citar este artículo: Cantillo-Medina CP, Sánchez-Castro LF, Ramírez-Guerrero AM, Muñoz-Bolaños MD, Quintero-Penagos HF, Cuero-Montaño SV. Calidad de vida y caracterización de las personas con Enfermedad Renal Crónica trasplantadas. Enferm Nefrol. 2021 Ene-Mar;24(1):83-92

\section{Resumen}

Introducción: La persona con enfermedad renal crónica trasplantada debe llevar a cabo una serie de autocuidados, debido a la complejidad del tratamiento y presencia de múltiples comorbilidades que unidos a factores de riesgo cardiovascular pueden afectar su calidad de vida.

Objetivo: Describir la calidad de vida en personas adultas con Enfermedad Renal Crónica trasplantadas y su relación con las características sociodemográficas y clínicas, en un grupo de pacientes atendidos en una institución de salud de alta complejidad, en Neiva-Colombia.

Material y Método: Estudio descriptivo de corte trasversal con 78 adultos que formaban parte del programa de trasplante, Neiva (Colombia). Se aplicaron los instrumentos: Ficha de Caracterización de la Persona con Enfermedad Crónica GCPC-UN-P versión 2014, y el Instrumento Calidad de Vida de Betty Ferrell.

Resultados: La dimensión con menor afectación fue la espiritual, seguida de la psicológica y física; la dimensión con mayor afectación fue la social. Los participantes presentaron percepción positiva de la calidad

\section{Correspondencia:}

Claudia Patricia Cantillo-Medina

E-mail: claudiacantillol@hotmail.com de vida en las dimensiones física, psicológica, social y espiritual; acorde al género presentaron mejor percepción las mujeres en las dimensiones psicológica, social y espiritual, mientras que en la dimensión física los hombres manifestaron una mejor percepción.

Conclusiones: Aspectos relacionados con la salud como independencia, función mental intacta, nivel educativo, apoyo familiar y vida laboral activa, favorecen el afrontamiento, aumentan la satisfacción y la calidad de vida de las personas trasplantadas.

PALABRAS CLAVE: calidad de vida; enfermedad renal crónica; trasplante renal; cuidado de enfermería; instrumento calidad de vida de Betty Ferrell.

\section{Quality of life and characterization of transplanted people for chronic kidney disease}

\section{Abstract}

Introduction: People transplanted for chronic kidney disease should carry out a series of self-care, due to the complexity of the treatment and the presence of multiple comorbidities that together with cardiovascular risk factors can affect their quality of life.

Objective: To describe the quality of life in adults transplanted for chronic kidney disease and the 
relationship with sociodemographic and clinical characteristics, in a group of patients treated in a highly complex health institution in Neiva-Colombia.

Material and Method: Descriptive cross-sectional study with 78 adults who were part of the transplant program, Neiva (Colombia). The characterization sheet of the person with chronic disease (GCPC-UN-P, version 2014), and the Betty Ferrell's quality of life instrument were applied.

Results: The dimension with the least affectation was the spiritual, followed by the psychological and physical. The most affected was social. The participants presented a positive perception of the quality of life in the physical, psychological, social and spiritual dimensions; According to gender, women had a better perception in the psychological, social and spiritual dimensions, while in the physical dimension, men showed a better perception.

Conclusions: Aspects related to health such as independence, intact mental function, educational level, family support and active work life, favor coping, increase satisfaction and quality of life of transplanted people.

KEYWORDS: quality of life; chronic kidney disease; kidney transplant; nursing care; Betty Ferrell quality of life instrument.

\section{Introducción}

La Enfermedad Renal Crónica (ERC) afecta al 10\% de la población en el mundo ${ }^{1}$, es considerada un problema de salud pública por sus crecientes tasas de incidencia y prevalencia en las últimas décadas; incremento asociado a la forma de vivir de las personas y el avance de la edad ${ }^{2-4}$. El progreso de esta condición produce deterioro de la función renal hasta que en estadios avanzados el individuo debe someterse a terapia de reemplazo renal diálisis o trasplante (TR) para restablecer y mantener el equilibrio del organismo, controlar la sintomatología de la uremia y prolongar la vida ${ }^{1}$.

Aunque el trasplante es la terapia de elección por la recuperación inmediata de la función del injerto, y la desaparición de muchos problemas adquiridos durante la $\mathrm{ERC}^{3}$, se ha de tener en cuenta que también habrá una gran necesidad de autocuidados debido a la complejidad del tratamiento y la presencia de comorbilidades, 5 , por lo que se han de asumir cambios en el estilo de vida, tomar múltiples medicamentos y manejar el estrés, aspectos que dificultan la adherencia al tratamiento $0^{7-8}$.

A pesar de la ostensible mejora que trae el TR, el individuo continua siendo un enfermo crónico, que puede experimentar sentimientos de incertidumbre, fatiga, dolor y cambios corporales por el tratamiento inmunosupresor9; además la susceptibilidad de desarrollar enfermedades como hipertensión, osteoporosis, alteraciones neurológicas y gastrointestinales, mayor predisposición a infecciones y obesidad. A este panorama se suma también el riesgo de muerte por evento cardiovascular, el cual se incrementa 10 veces en los pacientes trasplantados renales cuando se compara con personas sin insuficiencia renal ${ }^{5}$.

Los anteriores aspectos ocasionan pérdida en la autonomía y afectan de manera importante la vida de las personas ${ }^{10-12}$, convirtiendo el cuidado en un reto para el individuo, familia y personal de salud.

Se planteó como objetivo del estudio: Describir la calidad de vida en personas con ERC trasplantadas y su relación con las características sociodemográficas y clínicas en un grupo de pacientes atendidos en una institución de salud de alta complejidad en Neiva-Colombia.

\section{Material y Método}

Estudio descriptivo de corte transversal, realizado entre noviembre de 2018 y febrero de 2019 en el Hospital Hernando Moncaleano Perdomo, en Neiva-Colombia.

Se incluyeron a todos los pacientes mayores de 18 años trasplantados que dieron su consentimiento para participar en el mismo, quienes cumplieron con los criterios de inclusión de: Residir en la zona urbana de Neiva, y asistente regular a control periódico en el programa de trasplante, y como criterios de exclusión: persona trasplantada en tránsito de otra unidad renal, encontrarse en fase aguda de trasplante y/o con alteraciones cognitivas o conductuales.

Se recogieron variables sociodemográficas, funcionalidad, estado cognitivo así como calidad de vida percibida.

La recogida de datos se realizó en el domicilio o en la institución de salud, donde se informó a los participantes de los objetivos y procedimientos a realizar. 
Como instrumento para la recolección de la información se empleó la Ficha de Caracterización de la Persona con Enfermedad Crónica elaborada por el Grupo de cuidado al paciente crónico, Facultad de Enfermería, Universidad Nacional de Colombia (GCPC-UN-P) y validada en el contexto colombiano por Barrera Ortiz et al. en $2014^{13}$. Este instrumento está compuesto por 22 ítems organizados en tres categorías que identifican:

Dimensión 1. Las condiciones del paciente y su perfil sociodemográfico, midiendo la funcionalidad y estado cognitivo con las escalas PULSES ${ }^{14}$ y $\mathrm{SPMSQ}^{15}$.

Dimensión 2. La percepción de carga y de apoyo del paciente.

Dimensión 3. La utilización de los medios de información y comunicación (TICs).

Para valorar la calidad de vida se empleó el instrumento Calidad de Vida en Enfermedad Crónica versión paciente de Betty Ferrel (Instrumento Quality of life Q0L $)^{16}$, instrumento validado y adaptado por el grupo de cuidado al paciente crónico y la familia de la Universidad Nacional de Colombia. EI QOL cuenta con un nivel de confiabilidad de 0,89 y 0,69 de consistencia interna. Está compuesto por 41 ítems que abordan las dimensiones física, psicológica, social y espiritual de la calidad de vida, donde puntuaciones menores en las dimensiones físicas, espirituales y sociales están asociadas con mayor bienestar, por el contrario en la dimensión psicológica una mayor puntuación está asociada con mayor bienestar.

Para establecer la calidad de vida del individuo en cada una de las dimensiones, se sumaron las puntuaciones obtenidas en cada ítem de esa dimensión, posteriormente se calculó la diferencia con respecto al máximo bienestar en cada una de las dimensiones, determinándose el promedio muestral de las dos variables (promedio general y la diferencia de las dimensiones).

Para el análisis estadístico se llevó a cabo el estudio de la normalidad de las variables, presentándose las variables cuantitativas como media y desviación estándar; las variables cualitativas se presentan como frecuencias absolutas y relativas. Para la comparación de las diferentes categorías de las variables socioeconómicas con el resultado promedio de bienestar en las subescalas de calidad de vida, se utilizó la prueba de Kruskal-Wallis o ANOVA según correspondiera, considerándose relaciones estadísticamente significativas valores de $p<0,05$.
Los cálculos se llevaron a cabo mediante el paquete estadístico Stata 14.

La investigación fue aprobada por el Comité de Ética de la Universidad Surcolombiana, acta número 009 del 24 de octubre de 2018.

\section{Resultados}

Se incluyeron un total de 78 pacientes (48 varones), con una edad media de 49,4+13,01 años. En la Tabla 1 se muestra que la mayoría contaba con escolaridad primaria o secundaria, dedicados al trabajo independiente o al hogar, profesaban religión católica, residían en área urbana, con pareja, pertenecían a un nivel socioeconómico bajo y contaban con apoyo familiar. En cuanto a comorbilidades, la mayor parte presentaban entre 0 y 1 comorbilidad asociada, siendo esta la hipertensión arterial (HTA), glomerulonefrítis, lupus eritematoso sistémico o diabetes mellitus (DM).

En la Tabla 2 se observa la puntuación promedio en cada una de las dimensiones que componen el QOL. En la Tabla 3 se visualiza el porcentaje de puntuación de los ítems, por cada una de las dimensiones del QOL, destacando que la dimensión con menor afectación fue la espiritual, seguida de la psicológica y física mientras que la dimensión con mayor afectación fue la social. Los participantes presentaron percepción positiva de la calidad de vida en las dimensiones física, psicológica, social y espiritual; acorde al género presentaron mejor percepción las mujeres en las dimensiones psicológica, social y espiritual, mientras que los hombres manifestaron una mejor percepción en la dimensión física.

La relación entre las dimensiones de la calidad de vida y las características sociodemográficas se muestra en la Tabla 4 y la relación entre las dimensiones de la calidad de vida y las condiciones clínicas de las personas trasplantadas se observan en la Tabla 5.

\section{Discusión}

Este estudio pretende describir la calidad de vida de un grupo personas con ERC trasplantadas y su relación con las características sociodemográficas y clínicas. La edad avanzada de muchos participantes está en consonancia con el deterioro de la función renal como proceso fisiológico del envejecimiento, acelerado con la presencia 
Tabla 1. Caracterización sociodemográfica y clínica de los pacientes $(n=78)$.

\begin{tabular}{|c|c|c|c|}
\hline Variables & Categorías & $\mathbf{n}$ & Porcentaje (\%) \\
\hline \multirow[t]{4}{*}{ Grupos de edad } & Adolescencia (12-18 años) & 1 & 1,3 \\
\hline & Juventud (19-26 años) & 6 & 7,7 \\
\hline & Adultez (27-59 años) & 53 & 67,9 \\
\hline & Adulto Mayor (60 años y más) & 18 & 23,1 \\
\hline \multirow[t]{2}{*}{ Género } & Femenino & 30 & 38 \\
\hline & Masculino & 48 & 62 \\
\hline \multirow[t]{4}{*}{ Grado de escolaridad } & Primaria & 30 & 38 \\
\hline & Secundaria & 32 & 41 \\
\hline & Superior & 13 & 17 \\
\hline & Técnica o tecnológica & 3 & 4 \\
\hline \multirow[t]{4}{*}{ Ocupación } & Empleado(a) & 7 & 9 \\
\hline & Estudiante & 3 & 4 \\
\hline & Hogar & 32 & 41 \\
\hline & Trabajo independiente & 36 & 46 \\
\hline \multirow[t]{5}{*}{ Religión } & Católica & 62 & 79 \\
\hline & Evangélica & 1 & 1 \\
\hline & Cristiana & 6 & 8 \\
\hline & Panteísmo & 1 & 1 \\
\hline & Ninguna & 8 & 11 \\
\hline \multirow[t]{2}{*}{ Zona de residencia } & Rural & 10 & 13 \\
\hline & Urbana & 68 & 87 \\
\hline \multirow[t]{5}{*}{ Estado civil } & Casado(a) & 40 & 51 \\
\hline & Separado(a) & 2 & 3 \\
\hline & Soltero(a) & 20 & 26 \\
\hline & Unión libre & 15 & 19 \\
\hline & Viudo(a) & 1 & 1 \\
\hline \multirow[t]{2}{*}{ Niveles socioeconómicos } & Bajo & 74 & 94,87 \\
\hline & Medio & 4 & 5,13 \\
\hline \multirow[t]{4}{*}{ Tipos de apoyos recibidos } & Familiar & 76 & 98 \\
\hline & Económico & 71 & 92 \\
\hline & Psicológico & 66 & 85 \\
\hline & Social & 66 & 85 \\
\hline \multicolumn{4}{|c|}{ Características clínicas } \\
\hline \multirow[t]{2}{*}{$\begin{array}{l}\text { PULSES total } \\
\text { (nivel de dependencia) }\end{array}$} & $6-8$ & 74 & 95 \\
\hline & $9-11$ & 4 & 5 \\
\hline \multirow[t]{3}{*}{ SPQM (función mental) } & $0-2$ & 74 & 95 \\
\hline & $3-4$ & 3 & 4 \\
\hline & $5-6$ & 1 & 1 \\
\hline \multirow[t]{3}{*}{ Comorbilidades } & $0=$ Ninguna comorbilidad & 30 & 38,46 \\
\hline & $1=\mathrm{HTA}, \mathrm{DM}$ u otras. & 36 & 46,15 \\
\hline & $2=$ HTA y DM & 12 & 15,38 \\
\hline
\end{tabular}

$\mathbf{0}=$ Enfermedad renal crónica sin ninguna comorbilidad.

$\mathbf{l}=$ Enfermedad renal crónica con una comorbilidad diabetes mellitus (DM), hipertensión arteiral (HTA), glomerulonefrítis o lupus eritematoso sistémico.

2= Enfermedad renal crónica con dos comorbilidades HTA Y DM. de factores de riesgo asociados a estilos de vida inadecuados ${ }^{4,9}$. Como en otras series el género es predominante masculino, y la escolaridad primaria y secundaria, circunstancia que favorece un control inadecuado de patologías precursoras de enfermedades crónicas ${ }^{17}$, posiblemente porque la persona no entiende bien la enfermedad, el manejo en relación con la dieta a seguir y tratamiento farmacológico ${ }^{18}$; pues se espera que en las personas haya un aumento de la capacidad para cuidar de sí mismas cuando tienen mayor educación y por tanto mayor comprensión de su situación ${ }^{19}$.

Los participantes residían principalmente en un área urbana, pertenecientes al nivel socioeconómico bajo, dedicados al trabajo independiente y labores del hogar, contexto que los ubica en posición de vulnerabilidad económica ${ }^{20,21}$ puesto que gastos derivados de su situación no son cubiertos por el Sistema Publico ${ }^{22}$ lo que afecta la función social, económica y emocional de la perso$n a$, familia y entorno ${ }^{23}$. En cuanto al estado civil sobresalieron las personas con pareja es decir relacionando su ciclo vital con la familia ${ }^{24}$, aspecto que contribuye a superar los obstáculos y desafíos impuestos por las limitaciones físicas, tal y como lo plantea Costa ${ }^{25}$.

Los apoyos recibidos están dados por la familia y por la religión profesada (católica), los cuales según Silva ${ }^{26}$, favorecen el afrontamiento pues se crea un efecto protector, mejor tratamiento, adherencia a la medicación y nutrición, mejores resultados clínicos, que aumentan la satisfacción y calidad de vida relacionada con la salud. Estos hallazgos concuerdan con los resultados de otras investigaciones $5,9,21,27-30$.

Las comorbilidades más prevalentes en la población estudiada fueron la hipertensión arterial y la diabetes, datos concordantes con los reportados por Pérez et al. ${ }^{9}$. La mayoría de los participantes presentaron una percepción positiva de la calidad de vida significativamente superior en la dimensión física, por mejoría de las funciones orgánicas ocurrida después del TR, hallaz- 
Tabla 2. Promedio, percepción global en las dimensiones de la calidad de vida de las personas con trasplante renal $(n=78)$.

\begin{tabular}{|l|c|c|c|}
\hline Variable & Media & $\begin{array}{c}\text { Desviación } \\
\text { Estándar }\end{array}$ & $\begin{array}{c}\text { Diferencia } \\
\text { promedio }\end{array}$ \\
\hline Física & 15,01 & 36,07 & 37,41 \\
\hline Psicológica & 49,45 & 16,21 & 27,32 \\
\hline Social & 17,85 & 35,35 & 62,24 \\
\hline Espiritual & 20,63 & 13,88 & 19,13 \\
\hline
\end{tabular}

gos similares a los estudios de Antunes et al. ${ }^{31}$ y Villeneuve ${ }^{32}$. Las mujeres y adolescentes obtuvieron mejor percepción positiva en la mayoría de las dimensiones, a diferencia de otros autores ${ }^{33}$.

Las personas quienes presentaron bajo nivel de dependencia y función mental intacta obtuvieron un mejor bienestar físico, psicológico y social, siendo análogo con lo reportado por Carrillo-Algarra et al. ${ }^{34}$, donde se describe el trasplante como una esperanza de vida

Tabla 3. Porcentaje de puntuación de los ítems por dimensiones del instrumento Calidad de Vida de las Personas Trasplantadas ( $n=78$ ).

\begin{tabular}{|c|c|c|c|c|}
\hline & & Ítems & $\begin{array}{c}\text { Ningún } \\
\text { Problema \% }\end{array}$ & $\begin{array}{c}\text { Mayor } \\
\text { Alteración \% }\end{array}$ \\
\hline 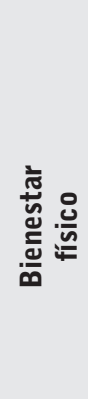 & $\begin{array}{l}1 . \\
2 . \\
3 . \\
4 . \\
5 . \\
6 . \\
7 . \\
8 .\end{array}$ & $\begin{array}{l}\text { Hasta qué punto es un problema para usted, sentir lo siguiente } \\
\text { Fatiga/agotamiento } \\
\text { Cambios de apetito } \\
\text { Dolor (es) } \\
\text { Cambios de sueño } \\
\text { Estreñimiento } \\
\text { Nausea/asco } \\
\text { Cambios en el sistema reproductivo (menstruación, fertilidad, impotencia) } \\
\text { Clasifique su salud física en general }\end{array}$ & $\begin{array}{l}48,7 \\
44,9 \\
55,1 \\
56,4 \\
73,1 \\
73,1 \\
61,5 \\
50,0\end{array}$ & $\begin{array}{l}9,0 \\
5,1 \\
2,6 \\
6,4 \\
3,8 \\
2,6 \\
6,4 \\
1,3\end{array}$ \\
\hline 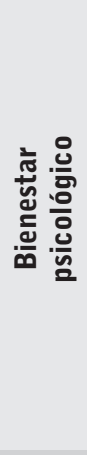 & $\begin{array}{l}9 . \\
10 . \\
11 . \\
12 . \\
13 . \\
14 . \\
15 . \\
16 . \\
17 .\end{array}$ & $\begin{array}{l}\text { Que tan difícil es para usted enfrentar/lidiar con su vida } \\
\text { como resultado de su enfermedad y tratamiento. } \\
\text { Que tan buena es su calidad de vida } \\
\text { Cuanta felicidad siente usted } \\
\text { Usted siente que tiene control de las cosas en su vida } \\
\text { Que tan satisfecho esta con su vida } \\
\text { Como clasificaría su capacidad para concentrarse o recordar cosas } \\
\text { Que tan útil se siente } \\
\text { Ha causado su enfermedad cambios en su apariencia } \\
\text { Ha causado su enfermedad o tratamiento cambios en su auto-concepto } \\
\text { (la manera en que usted se percibe/se ve) }\end{array}$ & $\begin{array}{l}30,8 \\
55,1 \\
48,7 \\
57,7 \\
71,8 \\
35,9 \\
55,1 \\
20,5 \\
25,6\end{array}$ & $\begin{array}{r}6,4 \\
0,0 \\
0,0 \\
0,0 \\
0,0 \\
1,3 \\
0,0 \\
25,6 \\
19,2\end{array}$ \\
\hline \multirow{2}{*}{ 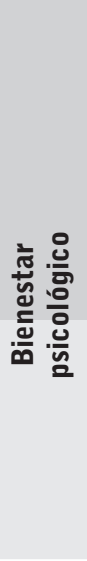 } & $\begin{array}{l}18 . \\
19 . \\
20 . \\
21 . \\
22 .\end{array}$ & $\begin{array}{l}\text { Cuanta aflicción/angustia le ocasiono los siguiente aspectos } \\
\text { de su enfermedad y tratamiento } \\
\text { El diagnóstico inicial } \\
\text { Los tratamientos para su enfermedad } \\
\text { El tiempo desde que su tratamiento termino } \\
\text { Cuanta ansiedad/desesperación tiene } \\
\text { Cuanta depresión/decaimiento siente usted }\end{array}$ & $\begin{array}{l}16,7 \\
21,8 \\
29,5 \\
34,6 \\
46,2\end{array}$ & $\begin{array}{c}44,9 \\
19,2 \\
3,8 \\
3,8 \\
1,3\end{array}$ \\
\hline & $\begin{array}{l}23 . \\
24 . \\
25 \\
26 .\end{array}$ & $\begin{array}{l}\text { Hasta qué punto tiene miedo/temor a: } \\
\text { Futuros exámenes diagnósticos } \\
\text { un segundo diagnóstico de otra alteración } \\
25 \text {. Retroceso en su enfermedad } \\
\text { Entiende el retroceso de su enfermedad }\end{array}$ & $\begin{array}{l}38,5 \\
28,2 \\
25,6 \\
61,5\end{array}$ & $\begin{array}{c}15,4 \\
21,8 \\
34,6 \\
6,4\end{array}$ \\
\hline 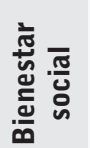 & $\begin{array}{l}27 . \\
28 .\end{array}$ & $\begin{array}{l}\text { Cuanta aflicción/angustia le ha ocasionado su enfermedad a su familia } \\
\text { Es suficiente el nivel de apoyo que usted recibe de parte de otros } \\
\text { para satisfacer sus necesidades }\end{array}$ & $\begin{array}{l}10,3 \\
52,6\end{array}$ & 33,3 \\
\hline
\end{tabular}


Tabla 3. Porcentaje de puntuación de los ítems por dimensiones del instrumento Calidad de Vida de las Personas Trasplantadas ( $\mathrm{n=78).}$

\begin{tabular}{|l|l|l|l|}
\hline & Ítems & $\begin{array}{c}\text { Ningún } \\
\text { Problema \% }\end{array}$ \\
Alteración $\%$
\end{tabular}

Tabla 4. Relación entre calidad de vida y las características sociodemográficas y condiciones clínicas de las personas trasplantadas ( $\mathrm{n=78}$ ).

\begin{tabular}{|c|c|c|c|c|}
\hline Variables & Dimensión Física & Dimensión psicológica & Dimensión Social & Dimensión Espiritual \\
\hline \multicolumn{5}{|l|}{ Edad } \\
\hline Adolescencia & 16 & 52 & 18 & 22 \\
\hline Juventud & 14,5 & 43,83 & 15,33 & 18,83 \\
\hline Adultez & 14 & 50,01 & 18,16 & 20,94 \\
\hline Adulto mayor & 14 & 49,5 & 17,72 & 20,22 \\
\hline$p$-value & 0,70 & 0,19 & 0,41 & 0,61 \\
\hline \multicolumn{5}{|l|}{ Género } \\
\hline Femenino & 14,5 & 51 & 18 & 20,05 \\
\hline Masculino & 14 & 48,47 & 18 & 20,5 \\
\hline p-value & 0,5 & 0,10 & 0,66 & 0,72 \\
\hline \multicolumn{5}{|l|}{ Escolaridad } \\
\hline Primaria & 15 & 49,06 & 18,73 & 19,96 \\
\hline Secundaria & 14 & 49,31 & 17,65 & 20,96 \\
\hline Técnica o Tecnológica & 18 & 49,33 & 15,66 & 21 \\
\hline Superior & 13 & 50,69 & 16,76 & 21,23 \\
\hline$p$-value & 0,27 & 0,91 & 0,32 & 0,72 \\
\hline \multicolumn{5}{|l|}{ Ocupación } \\
\hline Empleado(a) & 14 & 47,85 & 15,85 & 19,57 \\
\hline Estudiante & 14 & 44,33 & 14,33 & 17 \\
\hline Hogar & 15 & 49,96 & 18,40 & 20,06 \\
\hline Trabajo independiente & 13,5 & 49,72 & 18,02 & 21,63 \\
\hline$p$-value & 0,24 & 0,50 & 0,17 & 0,11 \\
\hline
\end{tabular}


Tabla 4. Relación entre calidad de vida y las características sociodemográficas y condiciones clínicas de las personas trasplantadas ( $n=78$ ).

\begin{tabular}{l|c|c|c|c|}
\hline Variables & Dimensión Física & Dimensión psicológica & Dimensión Social & Dimensión Espiritual \\
\hline Estado Civil & & & & \\
Casado(a) & 14,5 & 49,57 & 18,12 & 20,62 \\
Separado(a) & 13,5 & 45 & 14,5 & 18 \\
Soltero(a) & 14 & 48,65 & 16,9 & 20,9 \\
Unión libre & 12 & 50,4 & 18,6 & 20,93 \\
p-value & 0,51 & 0,71 & 0,40 & 0,67 \\
Nivel socioeconómico & & & & \\
Bajo & 13,33 & 48,20 & 17,46 & 19,88 \\
Medio & 15,5 & 50,75 & 15,25 & 20,5 \\
p-value & 0,15 & 0,10 & 0,11 & 0,28 \\
\hline
\end{tabular}

Tabla 5. Relación entre calidad de vida y las condiciones clínicas de las personas trasplantadas $(n=78)$.

\begin{tabular}{|c|c|c|c|c|}
\hline Variables & Dimensión Física & Dimensión psicológica & Dimensión Social & Dimensión Espiritual \\
\hline \multicolumn{5}{|c|}{$\begin{array}{l}\text { PULSES total } \\
\text { (nivel de dependencia) }\end{array}$} \\
\hline 6 a 8 & 14 & 49,75 & 17,82 & 20,81 \\
\hline 9 a 11 & 16,5 & 43,75 & 18,25 & 17,25 \\
\hline$p$-value & 0,23 & 0,079 & 0,83 & 0,081 \\
\hline \multicolumn{5}{|c|}{ SPQM (función mental) } \\
\hline 0 a 2 & 14 & 49,74 & 17,91 & 20,77 \\
\hline 3 a 4 & 18 & 44,66 & 15,33 & 19,33 \\
\hline 5 a 7 & 18 & 42 & 20 & 14 \\
\hline$p$-value & 0,10 & 0,23 & 0,46 & 0,21 \\
\hline \multicolumn{5}{|c|}{ Comorbilidades } \\
\hline 0 & 15 & 51,23 & 18,07 & 21,13 \\
\hline 1 & 13 & 47,83 & 17,92 & 20,5 \\
\hline 2 & 17 & 49,83 & 17,08 & 19,75 \\
\hline $\mathrm{p}$-value & 0,099 & 0,12 & 0,76 & 0,58 \\
\hline
\end{tabular}

e independencia, este evita ir tres veces por semana a hemodiálisis o cumplir el horario para sus recambios. De igual manera, quienes trabajaban presentan mejor percepción de bienestar, así como los que cuentan con pareja, semejante a lo concluido por Medellín Olaya ${ }^{29}$ donde este tipo de apoyo es un predictor de mejor calidad de vida.

En el bienestar físico un porcentaje pequeño de personas refirieron como problema severo continuar sintiendo fatiga, cambios en el apetito y alteración del sueño, lo que no concuerda con lo encontrado por Mendonça et al. ${ }^{33}$, donde todos los aspectos de este dominio mejoraron notablemente con el TR: reducción de los síntomas (dolor y cansancio), menor dependencia de los tratamientos y mejoría en el patrón de sueño.
Aunque la percepción de la calidad de vida en la dimensión psicológica mejora en la persona después del $T R$, en conjunto el miedo/temor, la aflicción/angustia generada por el diagnóstico inicial y los tratamientos para la enfermedad por los futuros exámenes diagnósticos, la aparición de una segunda enfermedad y el retroceso de la misma; fueron considerados como un problema severo, reporte similar a los hallazgos de Sampaio et al..$^{35}$, quienes encontraron que el miedo relacionado a la pérdida del injerto fue uno de los principales estresores después del trasplante, especialmente en quienes mantenían recuerdos negativos sobre la diálisis, relataban más temor en perder el injerto y tener que convivir nuevamente con la máquina de hemodiálisis. 
Acorde a lo anterior, la enfermedad impacta simultáneamente en la familia del paciente presentándose como un evento que genera además de aflicción una carga económica para ambos, indicadores que afectan negativamente en la persona trasplantada ${ }^{29}$. Recuperar la capacidad para trabajar y volver a las actividades sociales son aspectos que contribuyen a una mejor percepción ${ }^{33}$.

Las personas que se someten a un TR enfrentan procesos de adaptación intensos causados por la necesidad de cuidados complejos que pueden conducir a cambios psicológicos con alta complejidad y variabilidad emocional $^{33}$. En su estado de resiliencia buscan apoyo en su espiritualidad, una situación que para Medellín et al.29, juega un papel trascendental al agrupar las ideas filosóficas acerca de la vida y su propósito, pues tiene el poder de dar forma y significado al ser, reconociéndose en muchos casos como un componente amortiguador o de protección para la persona que cursa con la enfermedad y su familia, aspecto importante a tener en cuenta en esta población.

En cuanto a la relación entre las dimensiones de la calidad de vida, se encontró que la mayoría se relacionan significativamente entre ellas de manera positiva, además se observa conexión de la dimensión social con todas; mientras que la dimensión física solo presenta afinidad con la dimensión social. Estos datos pueden ser coherentes con la percepción de que a mayor bienestar físico, existe una mejor apreciación del bienestar social, a mayor bienestar social una mejor percepción de bienestar psicológico y espiritual. Lo descrito anteriormente puede afectar la calidad de vida, por consiguiente las condiciones adversas relacionadas con alguno de los bienestares debe ser abordado de manera integral.

Los resultados de esta investigación podrían servir de reflexión para continuar con el proceso de consolidación de programas desarrollados por equipos de salud expertos en el cuidado de las personas que viven en condición de cronicidad, con intervenciones orientadas a satisfacer las necesidades y requerimientos de los individuos con TR y sus familias, orientados a impactar en el bienestar de todas las dimensiones de la calidad de vida.

Este estudio no puede ser inferido a todas las personas trasplantadas, limitación debida al empleo de un muestreo por conveniencia. Sin embargo, se puede tener en cuenta para el cuidado y adecuación de las intervenciones enfermeras para la mejora de la calidad de vida de poblaciones con características similares.
Para la población estudiada se observó que la calidad de vida general es mayor cuando hay menos percepciones psicológicas negativas especialmente en la aflicción o angustia, por lo tanto se deben fortalecer programas de soporte permanente y se puede afirmar que los adultos trasplantados poseen recursos que favorecen el afrontamiento, aumentan la satisfacción y la calidad de vida relacionada con la salud.

\section{Recomendaciones}

Es pertinente continuar con estudios de investigación en Enfermería, donde se comparen diversos instrumentos utilizados en la evaluación de la calidad de vida según las diversas circunstancias sociales de cada país, lo que se facilitaría a través de programas estructurados de abordaje holístico a las personas trasplantadas y sus familias.

\section{Agradecimientos}

Agradecemos a las personas con ERC trasplantadas por su participación en este estudio, al Hospital Universitario Hernando Moncaleano Perdomo y a la Universidad Surcolombiana por el apoyo recibido.

Recepción: 21-11-20

Aceptación: 03-02-21

Publicación: 30-03-21

\section{Bibliografía}

1. Ayar Y, Ersoy A, Ocakoglu G, Yildiz A, Oruc A, Soyak $\mathrm{H}$ et al. Risk Factors Affecting Graft and Patient Survivals After Transplantation From Deceased Donors in a Developing Country: A Single-Center Experience. Transplant Proc. 2017;49(2):270-7.

2. Rebollo-Rubio A, Morales-Asencio JM, Pons-Raventos ME, Mansilla-Francisco JJ. Revisión de estudios sobre calidad de vida relacionada con la salud en la enfermedad renal crónica avanzada en España. Nefrologia. 2015;35(1):92-109. 
3. Mercado-Martínez FJ, Hernández-Ibarra E, Ascencio-Mera CD, Díaz-Medina BA, Padilla-Altamira C, Kierans C. Viviendo con trasplante renal, sin protección social en salud: ¿Qué dicen los enfermos sobre las dificultades económicas que enfrentan y sus efectos? Cad Saude Publica. 2014;30:2092-100.

4. Méndez-Durán $A$, Pérez-Aguilar $G$, Ayala-Ayala $F$, Ruíz-Rosas RA, González-Izquierdo J de J, Dávila-Torres J. Panorama epidemiológico de la insuficiencia renal crónica en el segundo nivel de atención del Instituto Mexicano del Seguro Social. Dial y Transpl. 2014;30(3):109-12.

5. Montoya-Hincapié SM, Paja-Becoche RM, Salas-Zapata C. Calidad de vida en pacientes trasplantados renales de una institución prestadora de servicios de salud en Medellín, Colombia. Univ y Salud. 2017;19:237.

6. Sistema Nacional de Salud. Documento Marco sobre Enfermedad Renal Crónica dentro de la Estrategia de Abordaje a la Cronicidad en el SNS. Ministerio de Sanidad Servicios Sociales e Igualdad. 2015. 54 p.

7. Palmer SC, Hanson CS, Craig JC, Strippoli GFM, Ruospo M, Campbell K, et al. Dietary and fluid restrictions in CKD: A thematic synthesis of patient views from qualitative studies. Am J Kidney Dis. 2015;65(4):559-73.

8. Pisano-González M M, González-Pisano A. La modificación de los hábitos y la adherencia terapéutica, clave para el control de la enfermedad crónica. Enferm Clin. 2014;24(1):59-66.

9. Pérez-Blancas C, Moyano-Espadero MC, Estepa-Del Árbol M, Crespo-Montero R. Factores asociados a calidad de vida relacionada con la salud de pacientes trasplantados de riñón. Enfermería Nefrológica. 2015;18(3):204-26.

10. Harwood L, Clark AM. Dialysis modality decision-making for older adults with chronic kidney disease. J Clin Nurs. 2014;23(23-24):3378-90.

11. Navarrete-Bonilla PC. Influencia de la Insuficiencia Renal Crónica en la Calidad de Vida de Pacientes Atendidos en la Clínica del Riñón Menydial, Tulcán 2016. Universidad Técnica del Norte; 2017.
12. Achury-Saldaña DM, Restrepo-Sanchez A, Torres-Castro NM, Buitrago-Mora $\mathrm{AL}$, Neira-Beltrán NX, Devia-Florez P. Competencia de los cuidadores familiares para cuidar a los pacientes con falla cardíaca. Rev Cuid. 2017;6(2):1721-32.

13. Barrera-Ortiz L, Vargas-Rosero E, Cendales PA. Encuesta de caracterización para el cuidado de una persona con enfermedad crónica. Investig en Enfermería Imagen y Desarrollo. 2014;17(1):2743.

14. Ward MJ, Lindeman CA, Public Health Service (DHEW) VA. Div. of Nursing. A. Instruments for Measuring Nursing Practice and Other Health Care Variables: Volume I [and] Volume 2. 1979; [Consultado: http://proxy.queensu.ca/login?url=http://search.ebscohost.com/login.aspx?direct=true \&db=eric\&AN=ED171763\&site=ehost-live, 1979].

15. Pfeiffer E (1975). Pfeiffer Short Portable Mental Status Questionnaire (SPMSQ):6-8.

16. Corredor-Parra LM, Mabel-Carrillo G. Validez y confiabilidad del instrumento de calidad de vida de Betty Ferrell, para personas con enfermedad crónica. Pontificia Universidad Javeriana Colombia 2016;129-48. [Consultado: ene-jun 2016]. Disponible en: http://www.redalyc.org/articulo. oa? $\mathrm{id}=145243501009$.

17. Carrillo-Algarra AJ. Análisis de la capacidad de autocuidados en pacientes en diálisis peritoneal. Enfermería Nefrológica. 2015;18(1):31-40.

18. Plantinga LC, Johansen $K L$, Schillinger $D$, Powe NR. Lower socioeconomic status and disability among US adults with chronic kidney disease, 1999-2008. Prev Chronic Dis. 2012;9(1):1999-2008.

19. Pérez-Acuña C, Riquelme-Hernandez G, Scharage-Goldenberg J, Armijo-Rodriguez I. Relación entre calidad de vida y representación de enfermedad en personas con enfermedad renal crónica terminal en tratamiento con hemodiálisis. Enfermería Nefrológica. 2015;18(2):89-96.

20. Bona K, Dussel V, Orellana L, Kang T, Geyer R, Feudtner $C$, et al. Economic impact of advanced pediatric Cancer on families. J Pain Symptom Manage. 2014;47(3):594-603. 
21. Chaparro-Díaz L, Sánchez-Herrera B, Carrillo-González G. Encuesta de caracterización del cuidado de la diada cuidador familiar - persona con enfermedad crónica. Rev Cienc y Cuid. 2014;11(2):31-45.

22. Montoya-Restrepo LA, Montoya-Restrepo IA, Zabdiel-Ocampo J, Sánchez-Herrera B, Chaparro-Diaz L. Diseño y validación de la encuesta "costo financiero del cuidado de la enfermedad crónica". Rev Científica Salud Uninorte. 2017;33(3):355-62.

23. Varela DC, Gaurían-Parra M, Hincapié NA, Hincapié SP, Rodriguez K. Factores no tradicionales influyentes en la calidad de vida de los pacientes de hemodiálisis. Rev Colomb Nefrol. 2014;1(1):17-24.

24. Moratto-Vásquez NS, Zapata-Posada JJ, Messager T. Conceptualización de ciclo vital familiar: una mirada a la producción durante el periodo comprendido entre los años 2002 a 2015. CES Psicol. 2015;8(2):103-21.

25. Costa-Arruda GM, Pinheiro-Nascimiento MB, De Medeiros SM, Costa-De Oliveira RR, Cossi-Santos $M$. Calidad de vida en pacientes con insuficiencia renal crónica en hemodiálisiss. Enfermería Glob. 2016;15(43):59-73.

26. Da Silva SM, Braido NF, Ottaviani AC, Gesualdo GD, Zazzetta MS, Orlandi F de S. Social support of adults and elderly with chronic kidney disease on dialysis. Rev Lat Am Enfermagem. 2016;24(0).

27. Sampaio de Brito DC, Moregola de Paula A, dos Santos Grincenkov FR, Lucchetti G, Sanders-Pinheiro $H$. Análisis de los cambios y dificultades surgidas después del trasplante renal: una investigación cualitativa. Rev Lat Am Enfermagem. 2015;23(3):419-26.

28. Portilla-Merino PP, Preciado-Vásquez J. Calidad de vida de pacientes trasplantados renales atendidos en el Hospital José Carrasco Arteaga 20072016, Cuenca Ecuador. Repositorio institucional
Universidad de Cauca. 2017.

29. Medellin-Olaya J, Carrillo-Gonzalez GM. Soporte social percibido y calidad de vida de personas con enfermedad renal crónica sometidas a trasplante renal. Av en Enfermería. 2014;32(2):206-16.

30. Moreno-Rubio F, Mora-Villarroel SL, Castelblanco-Toro C, Molina-López C, Ortiz-Varela LA. Trastorno emocional en el paciente renal trasplantado. Enfermería Nefrológica 2016;19(2):147-53.

31. Antunes AV, Mota Sousa LM, Justo C, Ferrer J, Frade $F$, Severino SSP, et al. Assessment of the perceived quality of life of a kidney transplant patient. Enfermería Nefrológica. 2018;21(2):138-44.

32. Villeneuve $C$, Laroche $M L$, Essig $M$, Merville $P$, Kamar $\mathrm{N}$, Coubret $\mathrm{A}$, et al. Evolution and determinants of health-related quality-of-life in kidney transplant patients over the first 3 years after transplantation. Transplantation. 2016;100(3):640-7.

33. De Mendonça AE0, de Góes Salvetti M, Maia EMC, de Oliveira e Silva AC, de Vasconcelos Torres $G$. Analysis of the physical aspects of quality of life of kidney recipients. Rev da Esc Enferm. 2015;49(1):76-81.

34. Carrillo Algarra AJ, Moreno Rubio F, Milena Buitrago S. Enfermedad Renal Crónica y trasplante renal: experiencias y superación de una estudiante de medicina. Index de Enfermería. 2015,24(4):250-4.

35. Sampaio de Brito, DC, Cristina $D$, Maregola de Paula, A, Dos Santos Grincenkov, FR, Luchetti G, Sanders-Pinheiro H. Análisis de los cambios y dificultades surgidas después del trasplante renal: una investigación cualitativa. Rev. Latino-Am. Enfermagem. 2015;23(3):419-26.

Este artículo se distribuye bajo una Licencia Creative Commons Atribución-NoComercial 4.0 Internacional. https://creativecommons.org/licenses/by-nc/4.0/ 